Article

\title{
Proteomic Profiling of the Microsomal Root Fraction: Discrimination of Pisum sativum L. Cultivars and Identification of Putative Root Growth Markers
}

\author{
Claudia-Nicole Meisrimler ${ }^{1,2, *,+}$, Stefanie Wienkoop ${ }^{3, \dagger}$ and Sabine Lüthje ${ }^{1}$ \\ 1 Oxidative Stress and Plant Proteomics Group, Biocenter Klein Flottbek and Botanical Garden, \\ University of Hamburg, Ohnhorststraße 18, D-22609 Hamburg, Germany; s.luthje@botanik.uni-hamburg.de \\ 2 Plant-Microbe Interactions, Department of Biology, Utrecht University, Padualaan 8, \\ $3584 \mathrm{CH}$ Utrecht, The Netherlands \\ 3 Deptartment of Ecogenomics and Systems Biology, University of Vienna, Althanstrasse 14, \\ A-1090 Vienna, Austria; stefanie.wienkoop@univie.ac.at \\ * Correspondence: c.meisrimler@uu.nl; Tel.: +31-30-253-2550 (ext. 3355) \\ + These authors contributed equally to this work.
}

Academic Editor: Jacek R. Wisniewski

Received: 8 November 2016; Accepted: 9 February 2017; Published: 2 March 2017

\begin{abstract}
Legumes are a large and economically important family, containing a variety of crop plants. Alongside different cereals, some fruits, and tropical roots, a number of leguminosae evolved for millennia as crops with human society. One of these legumes is Pisum sativum L., the common garden pea. In the past, breeding has been largely selective on improved above-ground organs. However, parameters, such as root-growth, which determines acquisition of nutrients and water, have largely been underestimated. Although the genome of $P$. sativum is still not fully sequenced, multiple proteomic studies have been published on a variety of physiological aspects in the last years. The presented work focused on the connection between root length and the influence of the microsomal root proteome of four different pea cultivars after five days of germination (cultivar Vroege, Girl from the Rhineland, Kelvedon Wonder, and Blauwschokker). In total, 60 proteins were identified to have significantly differential abundances in the four cultivars. Root growth of five-days old seedlings and their microsomal proteome revealed a similar separation pattern, suggesting that cultivar-specific root growth performance is explained by differential membrane and ribosomal protein levels. Hence, we reveal and discuss several putative root growth protein markers possibly playing a key role for improved primary root growth breeding strategies.
\end{abstract}

Keywords: Pisum sativum; microsomes; cultivar comparison; root morphology

\section{Introduction}

Pea cultivation developed together with human society for about 11,000 years [1]. Nowadays, Pisum sativum L. is one of the economical important legumes, including Glycine max (L.) MERR. (soybean), Phaseolus L. (bean), Cicer arietinum L. (chickpea), and Arachis hypogaea L. (peanut). In general, they are consumed in different forms. Peas are a rich source for proteins $(23 \%-25 \%)$ and essential amino acids, complex carbohydrates, and minerals, like iron, calcium, and potassium. In the most recent trends, pea seedlings are used to produce protein concentrates for protein powders as an alternative to dairy-derived whey proteins or glycine proteins. According to FAOSTAT data world production of the garden pea has more than quadrupled in the last 30 years [2]. The pea has been investigated as a model with respect to several physiological aspects and, interestingly enough, mapping the pea genome has lagged behind other crops because it has such a large and complex genome [3]. Aside from this, databases with information on pea genomic markers, genetic maps, quantitative trait loci (QTLs), 
and others are available on the coolseasonfoodlegume database, which is also usable for proteomic analysis [4].

Many proteomic studies concerning abiotic and biotic stress factors have been published in recent years [5-7]. Proteomic approaches in cultivar analysis, especially for orphan species, are an alternative to transcriptomics or genomics, and directly examine alterations in protein profiles. Protein quantitative trait loci (PQTLs), as used in medical applications [8], might be an alternative for cultivar analysis in the future and be of importance for production of stress tolerant and resistant pea cultivars. The use of proteomic approaches to analyse pea increased rigorously in recent years [9-12]. Generally, pea cultivars differ clearly in morphological and agronomic traits, in physiological and biochemical characteristics, and in their genomic structure. However, the proteins and genes responsible for these differences remain poorly characterized. Therefore, identifying cultivar-dependent differences of protein levels and composition are of interest for different agricultural applications. Roots are the major site of nutrient and water uptake and transport processes, therefore, they are of major interest for improved cultivars regarding mineral shortages, waterlogging, heavy metal pollution, and others. Furthermore, root growth is an important component of plant growth, but has received little attention by plant breeders due to difficulties associated with root observation. Improved root-related traits are likely to improve pea production, as well [12]. In the past, root length and root density have been observed for different abiotic stress factors, like phosphate starvation [13]. Root density is a far more complex trait than root length, making it more complicated to link proteomic datasets to this trait; therefore, we decided to focus first on root length in seedlings, rather than root density at later developmental stages.

The microsomal fraction, which consists mainly of membrane and ribosomal proteins, was frequently used in the past for proteomic approaches [14]. They contain an enriched amount of membrane proteins, relevant in root elongation growth, hormone receptors (e.g., ABP1), signal transduction, transport, and others, making them specifically interesting for root growth-related traits [15]. Changes in membrane proteins can often only be followed by proteomic approaches or Western blotting due to a mismatch of the transcriptional level to protein amounts [16]. In the present study we used four pea cultivars (Pisum sativum L. var. axiphium cultivar Vroege (Vroege), Pisum sativum L. var. sativum cultivar Girl from the Rhineland (GftR), Pisum sativum L. var. medullare cultivar Kelvedon Wonder (Kelvedon), and Pisum sativum L. var. sativum cultivar Blauwschokker (Blauwschokker) of different phenotypes. All four cultivars are traditionally cultivated peas in Europe, therefore, they are of interest for a broad group of applicants. According to the distributor their size is decreasing in the order Vroege $(80 \mathrm{~cm})>$ Blauwschokker $(60-80 \mathrm{~cm})>$ Kelvedon $(50-60 \mathrm{~cm})>$ Girl from the Rhineland $(20-30 \mathrm{~cm})$. The latter cultivar is a dwarf mutant with a defect in gibberellic acid biosynthesis. Cultivar specific protein profiles and protein abundance will be investigated in microsomal fractions. The correlation between root length and "marker proteins" will be discussed.

\section{Materials and Methods}

\subsection{Plant Material}

The four different European dish peas (cultivar Vroege, cultivar Girl from the Rhineland, cultivar Kelvedon Wonder, cultivar Blauwschokker; all cultivars were purchased from Sperli, Everswinkel, Germany) were cultivated for five days on paper. Seeds were washed with circulating tap water for $30 \mathrm{~min}$, submerged in $1 \% \mathrm{H}_{2} \mathrm{O}_{2}$ for $10 \mathrm{~min}$ and washed with $50 \%$ ethanol for $2 \mathrm{~min}$. After each disinfection step, seeds were rinsed with tap water. Germination was accomplished for five days in the dark at $26^{\circ} \mathrm{C}$.

\subsection{Microsomal Enrichment}

Roots (50 g fresh weight, 120-160 plants) were harvested and proteins extracted as described in Meisrimler et al. (2011) [6]. Membrane proteins were separated from the cell debris and soluble proteins by differential centrifugation $(10 \mathrm{~min}$ at $10,000 \times \mathrm{g}$ and $30 \mathrm{~min}$ at $50,000 \times \mathrm{g})$. 


\subsection{Washing Membranes}

The microsomal-enriched fraction was washed for $45 \mathrm{~min}$ at $4{ }^{\circ} \mathrm{C}$ with $20 \mathrm{mM}$ Tris- $\mathrm{HCl} \mathrm{pH}$ 7.6, $150 \mathrm{mM} \mathrm{KCl}, 150 \mathrm{mM}$ sucrose, $0.01 \%$ Triton X-100 and $1 \mathrm{mM}$ ethylenediaminetetraacetic acid (EDTA) to remove peripheral proteins and adsorbed soluble proteins as described in Meisrimler et al. [17]. Protein amounts were quantified as described by Bradford [18] in the presence of $0.01 \%$ Triton X-100 using bovine serum albumin as the standard.

\subsection{Sample Preparation for Mass Spectrometry}

Three independent microsomal preparations (for each cultivar) were used for the proteomic approach. Proteins were precipitated in chloroform/methanol 1:4 at $-20{ }^{\circ} \mathrm{C}$ overnight. Following centrifugation for $20 \mathrm{~min}$ at $12,000 \times g$ and $4{ }^{\circ} \mathrm{C}$, the pellet was washed with methanol three times. The pellet was resuspended in $200 \mathrm{mM} \mathrm{NH}_{4} \mathrm{HCO}_{3}$ (Merck, Darmstadt, Germany) pH 8.5 containing $8 \mathrm{M}$ urea and $10 \%$ acetonitrile. One hundred micrograms of protein for each replicate were reduced, alkylated, and pre-digested with LysC (Roche, Mannheim, Germany) for $6 \mathrm{~h}$ at $37^{\circ} \mathrm{C}$. Afterwards, the solution was diluted to $50 \mathrm{mM} \mathrm{NH}_{4} \mathrm{HCO}_{3} \mathrm{pH} 8.5,2 \mathrm{M}$ urea and $10 \%$ acetonitrile for digestion with trypsin beads (Applied Biosystems, Darmstad, Germany), which was carried out for $16 \mathrm{~h}$ at $37^{\circ} \mathrm{C}$. After removal of the beads by centrifugation, the supernatant was desalted using $\mathrm{C}_{18}$-columns, eluted with acetonitrile, and dried in a Speed Vac (Savant ISS110, Thermo Scientific, Bremen, Germany). Peptides were dissolved in $5 \%$ acetonitrile and $0.1 \%$ formic acid, and centrifuged again for $20 \mathrm{~min}$ at $12,000 \times g$ prior to LC-MS/MS analysis.

\subsection{Mass Spectrometry}

MS analysis was performed using an LTQ/Orbitrap XL (Thermo Scientific, Bremen, Germany) at a resolution of 30,000 $\times$, coupled to a 1D nano ultra Eksigent HPLC-System (Axel Semrau, Sprockhövel, Germany). Half a microliter of protein digest per sample was loaded on a $C_{18}$ column (75 $\mu$ m internal diameter, $15 \mathrm{~cm} \times 0.1 \mathrm{~mm}, 2.7 \mu \mathrm{m}$; Sigma-Aldrich, Vienna, Austria) which was used to separate the peptides. The mobile phases A ( $0.1 \%$ formic acid, $5 \%$ acetonitrile) and B $(0.1 \%$ formic acid, $80 \%$ acetonitrile) were used to form a gradient of $5 \%-60 \%$ acetonitrile in $60 \mathrm{~min}$. The flow rate was $400 \mathrm{~nL} \cdot \mathrm{min}^{-1}$.

Proteins were identified using the SEQUEST algorithm and the Proteome Discoverer (v 1.3, Thermo Scientific Inc.) to search MS data against an in-house fasta-file. The fasta-file was created as described previously [19] from downloads of different Pisum sativum sequence databases, fused from UniProt Uniref100 and a six frame translation of the Pisum_sativum_v1_Contig1005 (contig) Pisum sativum DB http:/ / www.coolseasonfoodlegume.org/ID56124, and PHVGI_Prot_rel4DFCI DB.

In silico peptide lists were generated with the following settings: trypsin as the digestion enzyme and a maximum of three missed cleavages. Mass tolerance was set to $5 \mathrm{ppm}$ for precursor ions and $0.8 \mathrm{Da}$ for fragment ions. Additionally, a decoy database containing reversed sequences was used to estimate the false discovery rate (FDR). Only high confidence (FDR $\leq 0.01 \%$ ) peptide identifications with a minimum XCorr of 2.0 and proteins with at least two distinct peptides were considered. The data matrix of the Proteome Discoverer (ThermoFisher Scientific, Darmstadt, Germany), containing spectral count information was used. Missing values were replaced by 0.5 (half of minimal value). For statistical analysis, a Student's $t$-test was carried out ( $p \leq 0.05$ for significance). For multivariate statistics (PCA), the MATLAB tool Covain was used [20]. A functional annotation was assigned using a Mapman mapping file, created using Mercator [21] on the basis of the in-house fasta-file assembled according to [22].

\subsection{Targeted Protein Marker Evaluation}

Several prototypic peptides of four mitochondrial membrane proteins (Supplemental Table S2) were selected for a targeted summed-intensity extraction using our ProtMax software (University of Vienna, Vienna, Austria) [23]. 


\section{Results}

\subsection{Morphological Changes}

All cultivars with typical phenotypes were shown in Figure 1A. Vroege showed significantly shorter roots than the other cultivars (Figure 1B), whereas cultivar Kelvedon exhibited the longest roots (Figure 1B). In general, even cultivar Kelvedon showed, significantly, the longest roots, a high variation in root length was observed in each cultivar, except for Vroege.

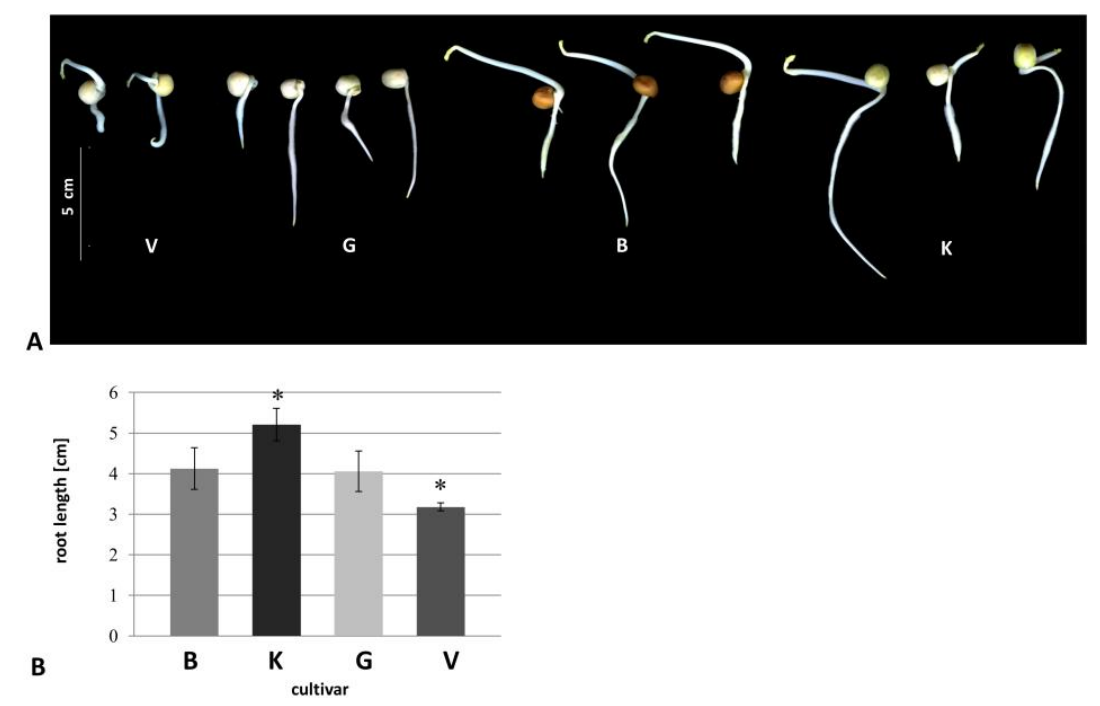

Figure 1. Five-days old seedlings. (A) Seedlings at day five; (B) Root length at day five. $\square$ Cultivar Blauwschokker (B), $\mathbf{\square}$ cultivar Kelvedon (K), cultivar GftR (G), $\square$ cultivar Vroege (V). Significant values $p \leq 0.05$ were marked with an asterisk. $n \geq 20$.

\subsection{Proteome Analysis}

\subsubsection{General Overview}

In total, 241 root proteins of a microsomal enrichment were identified from the LysC/tryptic in-solution digest by LC-MS (Supplemental Table S1). Among these were the standard marker of outer mitochondrial membrane (VDAC), inner mitochondrial membrane (cytochrome c oxidase), and endoplasmatic reticulum (KDEL-containing protein disulfide-isomerase). As shown in Supplemental Table S2, abundance of mitochondrial markers was similar in all cultivars, whereas that of the ER marker decreased from GftR > Vroege > Kelvedon > Blauwschokker. The levels of 60 proteins were changed differentially and statistically significant in the four cultivars at this developmental stage of the root (Table 1). PC1 (explaining 45\% of the discrimination), separated membrane proteins of the cultivars that seemed related to root growth; from Kelvedon, with the longest root growth, to a clustering of Blauwschokker and GftR, and then Vroege, with the smallest growth (Figures 1A and 2A).

The smallest number (20) of significantly-changed proteins was found between GftR and Kelvedon, and a large number (36) for e.g., Kelvedon against Vroege (Table 1). The differences between the latter cultivars were also underlined by the most distinct changes in the protein ratio.

Overall, 20\% of the significantly-changed proteins shown in Table 1 had a transmembrane region (Figure 2B). Most proteins identified to be of differential abundance were related to protein synthesis $(42 \%)$, protein folding $(7 \%)$ and amino acid metabolism $(7 \%)$, where this percentage is called $\%$ abundance (Figure 2C). In addition, $8.5 \%$ of the proteins with a significant difference between the cultivars could not be assigned to any specific function (Figure 2C). All significantly-different proteins were analysed for their subcellular localisation, using information for the corresponding protein entry in NCBI or Uniprot. If no data about localisation were available, PlantmPLOC was used to compute 
localisation. The highest amount of proteins identified appeared to be cytosolic origin (47\%), followed by mitochondria (19\%), and golgi (14\%) (Figure 2D).

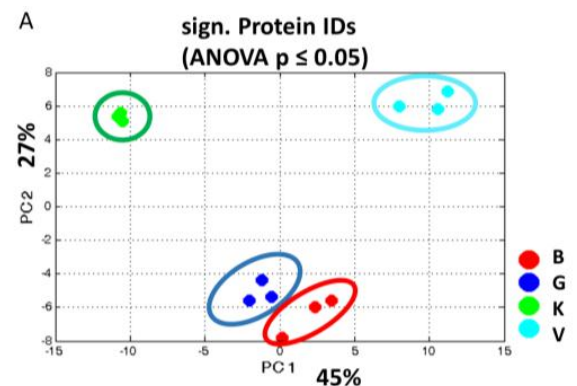

C

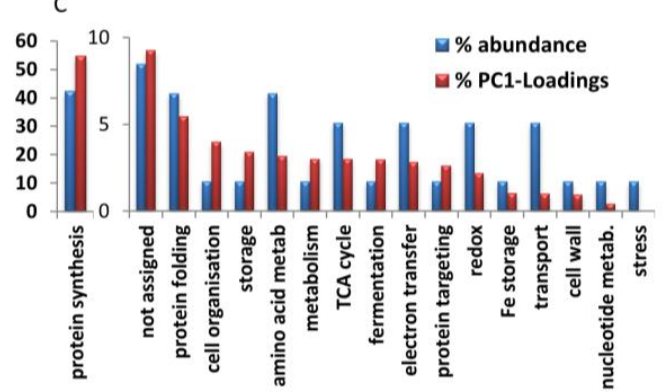

B
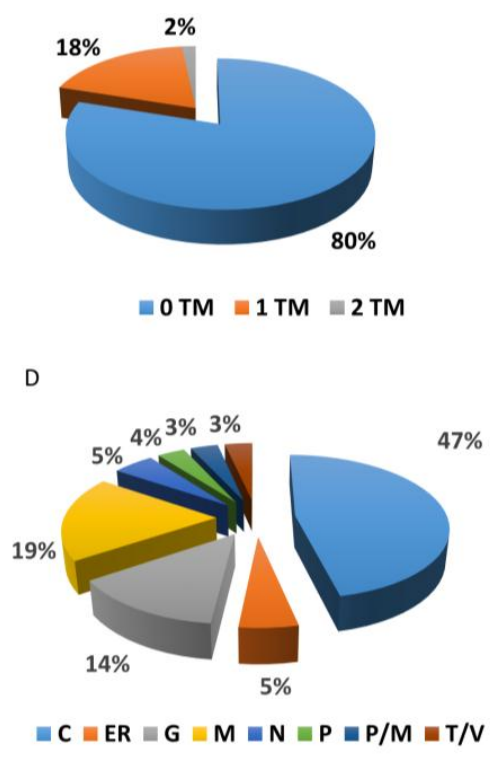

Figure 2. Comparison of the microsomal enriched and significantly different proteins across roots of four pea cultivars by shotgun LC-MS. MS analyses were performed using an LTQ/Orbitrap XL and significantly different $(t$-test, $p \leq 0.05)$ proteins were analysed in detail by PCA (A): B, Blauwschokker (red); G, GftR (blue); K, Kelvedon (green); V, Vroege (cyan). Significant proteins shown in Table 1 were analysed for transmembrane (TM) domains using HMMTOP (B), percentage of proteins corresponding to the functional groups in relation to their PC1 loadings (C), and cellular localisation of proteins: $\mathrm{M}$; mitochondria; P; plastid; G; golgi; N; nucleus; C; cytosol; T; tonoplast; V; vesicles (D).

\subsubsection{Cultivar Specific Differences—Proteins with the Highest Separation Impact on PC1}

The largest functional group of significantly-changed protein levels between the cultivars was protein synthesis, which also showed the highest percentage of PC1 loadings ( 55\%) (Figure $2 \mathrm{C}$ ). In total, six proteins of the $60 \mathrm{~S}$ ribosome and twelve of the $40 \mathrm{~S}$ ribosome were identified to be differentially abundant in the cultivars. All ribosomal proteins showed the significantly highest abundance in cultivar Vroege.

In contrast, the channel protein TOM40 homolog1—categorized to protein targeting-was clearly of highest abundance in Kelvedon compared to the other cultivars (up to 16.6-fold). This protein is most likely to be localized in the mitochondria outer membrane (Table 1).

Other protein groups that show a higher percentage of PC1 loadings (high impact on separation) compared to their abundance (in terms of numbers of proteins) were assigned to cell organisation, storage, metabolism, fermentation, as well as the group of unassigned proteins (Figure 2C).

For instance, convicilin, a major storage protein of pea, was eleven to 22-fold higher in cultivar Vroege. Furthermore, metabolism-related protein dihydrolipoamide dehydrogenase, the fermentation-related protein ADH family 2 member B7 protein and, most significantly, regarding both abundance and PC1 loading, annexin-like protein RJ4, located to the nucleus membrane, were mainly increased in Vroege (Table 1).

All other groups, such as amino acid metabolism, redox and electron transfer, cell wall and transport, showed only a low percentage on the impact of PC1 loadings, which was also comparably lower than their abundances and, thus, less likely related to root growth. 
Table 1. Significantly different proteins in the four cultivars identified by LC-MS. The heat map table shows all identified proteins that changed significantly in at least one of the cultivars. Each measurement consisted of three biological replicates and each biological replicate was a pool of 100 plants to keep variation of individuals low. Significant changes in ratios (Student's $t$-test $<0.05$; ratio $<0.5$ or ratio $>2.0$ ) are marked in bold. Decreased values are marked in green; increased values are marked in red. Numbering is given in the first column. Accession numbers and protein description correspond to the database used for identification. TM, transmembrane domains: TM1 was computed with TMHMM [24] and TM2 was computed with HMMTOP [25]. Cellular function of the proteins; based on MapMan and manual validation with InterProScan. Localization (Loc) is based on Uniprot or NCBI information and, if localization was unknown, marked with an asterisk. PlantMPloc was used for prediction [26-28]; localization: M; mitochondria; P; plastid; G; golgi; N; nucleus; C; cytosol; T; tonoplast; V; vesicles. Column 6-9, show the average spectral counts (SC). Columns 10-15, show the ratios for all possible cultivar comparisons. In the last column, PC1 loading is shown. Amount of significant fold changes: blue shades, decrease; red shades, increase. Statistical significant data for $p \leq 0.05$ are shown in bold. Abbreviations for the cultivars were chosen as follows: B; Blauwschokker; V; Vroege; G; Girl from the Rheinland, GftR; K; Kelvedon Wonder. Significant fold changes: blue shades, decrease; red shades, increase.

\begin{tabular}{|c|c|c|c|c|c|c|c|c|c|c|c|c|c|c|c|c|}
\hline \multirow{2}{*}{ Accession } & \multirow{2}{*}{ Description } & \multirow{2}{*}{ TM1 } & \multirow{2}{*}{ TM2 } & \multirow{2}{*}{ Function } & \multicolumn{5}{|c|}{$\mathrm{SC}$} & \multicolumn{6}{|c|}{ Ratio } & \multirow{2}{*}{$\begin{array}{c}\text { PC1 } \\
\text { Loadings }\end{array}$} \\
\hline & & & & & loc & B & $\mathrm{V}$ & G & $\mathrm{K}$ & $\mathrm{V} / \mathrm{K}$ & $\mathbf{B} / \mathbf{K}$ & G/K & V/G & V/B & G/B & \\
\hline contig12248 & Annexin-like protein RJ4 & 0 & 0 & cell organisation & $\mathrm{N}^{*}$ & 5.7 & 18.7 & 3.7 & 0.5 & 37.3 & 11.3 & 7.3 & 5.1 & 3.3 & 0.7 & 0.2490 \\
\hline p.sativum_wa1_contig21697 & 60 S ribosomal protein L4-1 & 0 & 0 & protein synthesis & $\mathrm{C}$ & 10.3 & 15.3 & 8.7 & 0.5 & 30.7 & 20.7 & 17.3 & 1.8 & 1.5 & 0.8 & 0.2379 \\
\hline contig10741 & Convicilin & 0 & 0 & storage & $\mathrm{G}^{*}$ & 1.0 & 11.7 & 0.5 & 0.5 & 23.3 & 2.0 & 1.0 & 23.3 & 11.1 & 0.5 & 0.2130 \\
\hline p.sativum_wa1_contig28348 & $40 \mathrm{~S}$ ribosomal protein $\mathrm{S} 8$ & 0 & 0 & protein synthesis & $\mathrm{C}$ & 3.2 & 8.0 & 1.7 & 0.5 & 16.0 & 6.3 & 3.3 & 4.8 & 2.5 & 0.5 & 0.2017 \\
\hline p.sativum_wa1_contig16856 & $60 \mathrm{~S}$ acidic ribosomal protein $\mathrm{P} 2-2$ & 0 & 0 & protein synthesis & $\mathrm{C}$ & 2.0 & 10.7 & 4.0 & 0.5 & 21.3 & 4.0 & 8.0 & 2.7 & 5.3 & 2.0 & 0.1961 \\
\hline contig17941 & Endoplasmin homolog & 0 & 0 & protein folding & ER & 2.5 & 9.0 & 6.0 & 0.5 & 18.0 & 5.0 & 12.0 & 1.5 & 3.6 & 2.5 & 0.1904 \\
\hline $9955324 \mathrm{pdb}$ & Dihydrolipoamide dehydrogenase & 0 & 0 & metabolism & $\mathrm{M}^{*}$ & 1.3 & 8.0 & 0.5 & 0.5 & 16.0 & 2.7 & 1.0 & 16.0 & 5.9 & 0.4 & 0.1878 \\
\hline Pisum_sativum_v2_Contig4072 & ADH family 2 member B7 & 0 & 1 & fermentation & $\mathrm{M}$ & 12.3 & 10.3 & 5.7 & 1.0 & 10.3 & 12.3 & 5.7 & 1.8 & 0.8 & 0.5 & 0.1850 \\
\hline contig02118 & 60S ribosomal protein L13-1 & 0 & 0 & protein synthesis & $\mathrm{C}$ & 1.7 & 6.7 & 0.5 & 0.5 & 13.3 & 3.3 & 1.0 & 13.3 & 4.0 & 0.3 & 0.1830 \\
\hline p.sativum_wa1_contig25888 & 40S ribosomal protein S4-2 & 0 & 0 & protein synthesis & $\mathrm{C}$ & 3.0 & 6.0 & 2.2 & 0.5 & 12.0 & 6.0 & 4.3 & 2.8 & 2.0 & 0.7 & 0.1766 \\
\hline Contig3080 & $40 \mathrm{~S}$ ribosomal protein $\mathrm{S} 17-4$ & 0 & 0 & protein synthesis & $\mathrm{C}$ & 4.0 & 5.0 & 0.5 & 0.5 & 10.0 & 8.0 & 1.0 & 10.0 & 1.3 & 0.1 & 0.1763 \\
\hline EX568861 & $40 \mathrm{~S}$ ribosomal protein $\mathrm{S} 17$ & 0 & 0 & protein synthesis & $\mathrm{C}$ & 4.0 & 5.0 & 0.5 & 0.5 & 10.0 & 8.0 & 1.0 & 10.0 & 1.3 & 0.1 & 0.1763 \\
\hline GH720005 & $60 \mathrm{~S}$ ribosomal protein $\mathrm{L} 9$ & 0 & 0 & protein synthesis & $\mathrm{C}$ & 5.0 & 6.3 & 5.0 & 0.5 & 12.7 & 10.0 & 10.0 & 1.3 & 1.3 & 1.0 & 0.1755 \\
\hline p.sativum_wa1_contig17528 & 60 S ribosomal protein L13-1 & 0 & 0 & protein synthesis & $\mathrm{C}$ & 1.7 & 5.3 & 1.3 & 0.5 & 10.7 & 3.3 & 2.7 & 4.0 & 3.2 & 0.8 & 0.1642 \\
\hline Pisum_sativum_v2_Contig5665 & $60 \mathrm{~S}$ ribosomal protein $\mathrm{L} 4$ & 0 & 0 & protein synthesis & $\mathrm{C}$ & 2.5 & 5.3 & 1.3 & 0.5 & 10.7 & 5.0 & 2.7 & 4.0 & 2.1 & 0.5 & 0.1621 \\
\hline p.sativum_wa1_contig24787 & 40S ribosomal protein S2-4 & 0 & 0 & protein synthesis & $\mathrm{C}$ & 1.5 & 5.3 & 0.5 & 0.5 & 10.7 & 3.0 & 1.0 & 10.7 & 3.6 & 0.3 & 0.1604 \\
\hline contig13719 & 40S ribosomal protein SA & 0 & 0 & protein synthesis & $\mathrm{C}$ & 0.5 & 6.0 & 0.5 & 0.5 & 12.0 & 1.0 & 1.0 & 12.0 & 12.5 & 1.0 & 0.1585 \\
\hline p.sativum_wa1_contig21882 & 60S ribosomal protein L4-1 & 0 & 0 & protein synthesis & $\mathrm{C}$ & 2.2 & 5.0 & 1.3 & 0.5 & 10.0 & 4.3 & 2.7 & 3.8 & 2.3 & 0.6 & 0.1570 \\
\hline p.sativum_wa1_contig07673 & 40 S ribosomal protein S15-4 & 0 & 0 & protein synthesis & $\mathrm{C}$ & 5.0 & 4.7 & 6.0 & 0.5 & 9.3 & 10.0 & 12.0 & 0.8 & 0.9 & 1.3 & 0.1541 \\
\hline p.sativum_wa1_contig22133 & $40 \mathrm{~S}$ ribosomal protein $\mathrm{SA}$ & 0 & 0 & protein synthesis & $\mathrm{C}$ & 0.5 & 5.3 & 0.5 & 0.5 & 10.7 & 1.0 & 1.0 & 10.7 & 11.1 & 1.0 & 0.1517 \\
\hline AC̄U20293.1 & $40 \mathrm{~S}$ ribosomal protein $\mathrm{S} 30$ & 0 & 0 & protein synthesis & $\mathrm{C}$ & 4.3 & 4.3 & 4.7 & 0.5 & 8.7 & 8.7 & 9.3 & 0.9 & 1.0 & 1.1 & 0.1486 \\
\hline ACJ85955.1 & 60S ribosomal L12-like protein & 0 & 0 & protein synthesis & $\mathrm{C}$ & 1.3 & 4.3 & 1.5 & 0.5 & 8.7 & 2.7 & 3.0 & 2.9 & 3.2 & 1.1 & 0.1429 \\
\hline BAB40231.1 & S-type apyrase & 0 & 1 & not assigned & $\mathrm{N}^{*}$ & 8.0 & 3.3 & 8.0 & 0.5 & 6.7 & 16.0 & 16.0 & 0.4 & 0.4 & 1.0 & 0.1345 \\
\hline p.sativum_wa1_contig03572 & $40 \mathrm{~S}$ ribosomal protein $\mathrm{S} 3 \mathrm{a}$ & 0 & 0 & protein synthesis & $\mathrm{C}$ & 0.5 & 3.7 & 0.5 & 0.5 & 7.3 & 1.0 & 1.0 & 7.3 & 7.1 & 1.0 & 0.1274 \\
\hline contig09310 & Uncharacterized protein At5g10860 & 0 & 0 & not assigned & $\mathrm{M}$ & 1.7 & 2.7 & 0.5 & 0.5 & 5.3 & 3.3 & 1.0 & 5.3 & 1.6 & 0.3 & 0.1241 \\
\hline
\end{tabular}


Table 1. Cont

\begin{tabular}{|c|c|c|c|c|c|c|c|c|c|c|c|c|c|c|c|c|}
\hline \multirow{2}{*}{ Accession } & \multirow{2}{*}{ Description } & \multirow{2}{*}{ TM1 } & \multirow{2}{*}{ TM2 } & \multirow{2}{*}{ Function } & \multicolumn{5}{|c|}{ SC } & \multicolumn{6}{|c|}{ Ratio } & \multirow{2}{*}{$\begin{array}{c}\text { PC1 } \\
\text { Loadings }\end{array}$} \\
\hline & & & & & loc & B & $\mathrm{V}$ & G & $\mathbf{K}$ & $\mathrm{V} / \mathrm{K}$ & $\mathrm{B} / \mathrm{K}$ & $\mathrm{G} / \mathrm{K}$ & V/G & $\mathrm{V} / \mathbf{B}$ & $\mathrm{G} / \mathrm{B}$ & \\
\hline Pisum_sativum_v2_Contig1288 & 40S ribosomal protein S3a & 0 & 0 & protein synthesis & $\mathrm{C}$ & 0.5 & 3.3 & 0.5 & 0.5 & 6.7 & 1.0 & 1.0 & 6.7 & 6.7 & 1.0 & 0.1209 \\
\hline Pisum_sativum_v2_Contig4787 & Protein disulfide isomerase-like 1-4 & 0 & 1 & redox & ER & 4.7 & 6.0 & 7.7 & 1.7 & 3.6 & 2.8 & 4.6 & 0.8 & 1.3 & 1.7 & 0.1198 \\
\hline Pisum_sativum_v2_Contig857 & 40S ribosomal protein $\mathrm{S} 18$ & 0 & 0 & protein synthesis & $\mathrm{C}$ & 0.5 & 3.0 & 0.5 & 0.5 & 6.0 & 1.0 & 1.0 & 6.0 & 5.9 & 1.0 & 0.1132 \\
\hline p.sativum_wa1_contig04121 & $\begin{array}{l}\text { LOX homol. domain-containing } \\
\text { protein } 1\end{array}$ & 0 & 0 & not assigned & $\mathrm{G}^{*}$ & 2.3 & 2.7 & 3.7 & 0.5 & 5.3 & 4.7 & 7.3 & 0.7 & 1.1 & 1.7 & 0.1119 \\
\hline Pisum_sativum_v2_Contig5583 & Succinyl-CoA ligase sub. $\mathrm{b}$ & 0 & 1 & TCA cycle & $\mathrm{G}^{*}$ & 0.5 & 5.3 & 0.5 & 1.3 & 4.0 & 0.4 & 0.4 & 10.7 & 11.1 & 1.0 & 0.1109 \\
\hline GH720468 & 40S ribosomal protein S25-2 & 0 & 0 & protein synthesis & $\mathrm{C}$ & 4.3 & 3.5 & 3.3 & 0.5 & 7.0 & 8.7 & 6.7 & 1.1 & 0.8 & 0.8 & 0.1089 \\
\hline Contig386 & 40S ribosomal protein $\mathrm{S} 14$ & 0 & 0 & protein synthesis & $\mathrm{C}$ & 11.3 & 15.0 & 12.0 & 4.7 & 3.2 & 2.4 & 2.6 & 1.3 & 1.3 & 1.1 & 0.0823 \\
\hline GH720846 & 60S ribosomal protein L22-2 & 0 & 0 & protein synthesis & $\mathrm{C}$ & 3.0 & 1.8 & 1.5 & 0.5 & 3.7 & 6.0 & 3.0 & 1.2 & 0.6 & 0.5 & 0.0781 \\
\hline contig09315 & Cysteine synthase & 0 & 0 & amino acid metab & G & 16.7 & 15.7 & 6.7 & 6.3 & 2.5 & 2.6 & 1.1 & 2.4 & 0.9 & 0.4 & 0.0702 \\
\hline AAB24082.1 & ferritin & 0 & 0 & Fe storage & $\mathrm{P}$ & 4.3 & 8.7 & 4.3 & 3.3 & 2.6 & 1.3 & 1.3 & 2.0 & 2.0 & 1.0 & 0.0643 \\
\hline contig21497 & Cysteine synthase & 0 & 0 & amino acid metab. & $\mathrm{P} / \mathrm{M}$ & 18.7 & 16.0 & 7.7 & 7.3 & 2.2 & 2.5 & 1.0 & 2.1 & 0.9 & 0.4 & 0.0628 \\
\hline Pisum_sativum_v2_Contig4779 & UDP-glucuronic acid decarboxylase 1 & 1 & 1 & cell wall & $\underset{*}{\mathrm{G} / \mathrm{N}}$ & 0.5 & 1.8 & 3.7 & 0.5 & 3.7 & 1.0 & 7.3 & 0.5 & 3.7 & 10.0 & 0.0584 \\
\hline contig20699 & NADH-Q oxidoreductase $40 \mathrm{kDa}$ sub. & 0 & 0 & electron transfer & $\mathrm{M}$ & 3.0 & 3.3 & 0.5 & 2.5 & 1.3 & 1.2 & 0.2 & 6.7 & 1.1 & 0.2 & 0.0532 \\
\hline p.sativum_wa1_contig18574 & Chaperonin CPN60-2 & 0 & 0 & protein folding & $\mathrm{M}$ & 17.7 & 18.7 & 7.3 & 10.3 & 1.8 & 1.7 & 0.7 & 2.5 & 1.1 & 0.4 & 0.0515 \\
\hline contig22493 & Cysteine synthase & 0 & 0 & amino acid metab & $\mathrm{G}$ & 18.7 & 10.0 & 5.3 & 6.3 & 1.6 & 2.9 & 0.8 & 1.9 & 0.5 & 0.3 & 0.0472 \\
\hline Pisum_sativum_v2_Contig6323 & $\begin{array}{l}\text { component of 2-oxoglutarate } \\
\text { dehydrogenase }\end{array}$ & 0 & 1 & TCA cycle & $G^{*}$ & 5.0 & 4.7 & 1.0 & 3.3 & 1.4 & 1.5 & 0.3 & 4.7 & 0.9 & 0.2 & 0.0455 \\
\hline p.sativum_wa1_contig18562 & Chaperonin CPN60-2 & 0 & 0 & protein folding & $\mathrm{M}$ & 14.3 & 17.7 & 5.3 & 11.0 & 1.6 & 1.3 & 0.5 & 3.3 & 1.2 & 0.4 & 0.0439 \\
\hline Pisum_sativum_v2_Contig1332 & Probable arginase & 1 & 1 & amino acid metab. & G & 4.3 & 8.0 & 11.3 & 5.3 & 1.5 & 0.8 & 2.1 & 0.7 & 1.9 & 2.5 & 0.0191 \\
\hline $189095946 \mathrm{pdb}$ & Mitochondrial Type Ii Peroxiredoxin & 0 & 0 & redox & $\mathrm{M}$ & 0.5 & 5.7 & 0.5 & 4.0 & 1.4 & 0.1 & 0.1 & 11.3 & 11.1 & 1.0 & 0.0130 \\
\hline p.sativum_wa1_contig02960 & Elongation factor 1-alpha & 0 & 0 & protein synthesis & $\mathrm{C}$ & 1.3 & 4.3 & 5.3 & 3.3 & 1.3 & 0.4 & 1.6 & 0.8 & 3.2 & 3.3 & 0.0026 \\
\hline P37900.585272 sp & HSP70 & 0 & 0 & stress & $\mathrm{M}$ & 0.5 & 6.0 & 0.5 & 5.3 & 1.1 & 0.1 & 0.1 & 12.0 & 12.5 & 1.0 & -0.0022 \\
\hline Pisum_sativum_v2_Contig5744 & $\begin{array}{c}\text { Probable protein } \\
\text { disulfide-isomerase A6 }\end{array}$ & 0 & 1 & redox & ER & 12.7 & 25.7 & 18.3 & 25.3 & 1.0 & 0.5 & 0.7 & 1.4 & 2.0 & 1.4 & -0.0040 \\
\hline Pisum_sativum_v2_Contig5499 & NADH-Q oxidoreductase subunit & 0 & 1 & electron transfer & $\mathrm{G}^{*}$ & 5.0 & 7.7 & 2.5 & 9.3 & 0.8 & 0.5 & 0.3 & 3.1 & 1.5 & 0.5 & -0.0090 \\
\hline G9JKP3 & Plastid OEP 16.2 & 0 & 2 & transport & $\mathrm{P}$ & 0.5 & 5.7 & 2.5 & 5.7 & 1.0 & 0.1 & 0.4 & 2.3 & 11.1 & 5.0 & -0.0138 \\
\hline CBD35496.1 & Elongation factor 1-alpha & 0 & 0 & protein synthesis & $\mathrm{C}$ & 0.5 & 3.7 & 4.0 & 3.3 & 1.1 & 0.2 & 1.2 & 0.9 & 7.1 & 10.0 & -0.0179 \\
\hline p.sativum_wa1_contig06534 & V-type proton ATPase 116 kDa sub.a 1 & 0 & 0 & transport & $\mathrm{T} / \mathrm{V}$ & 3.0 & 0.5 & 0.5 & 1.3 & 0.4 & 2.3 & 0.4 & 1.0 & 0.2 & 0.2 & -0.0201 \\
\hline p.sativum_wa1_contig17185 & Elongation factor 1-alpha & 0 & 0 & protein synthesis & $\mathrm{C}$ & 0.5 & 3.0 & 2.5 & 3.0 & 1.0 & 0.2 & 0.8 & 1.2 & 5.9 & 5.0 & -0.0217 \\
\hline $56554368 \mathrm{pdb}$ & Nucleoside diphosphate kinase & 0 & 1 & not assigned & $\mathrm{M}$ & 1.0 & 4.0 & 2.5 & 5.0 & 0.8 & 0.2 & 0.5 & 1.6 & 4.0 & 2.5 & -0.0246 \\
\hline p.sativum_wa1_contig12891 & Adenylate kinase B & 0 & 0 & nucleotide metab. & $\mathrm{C}$ & 0.5 & 8.3 & 4.0 & 8.0 & 1.0 & 0.1 & 0.5 & 2.1 & 16.7 & 10.0 & -0.0259 \\
\hline p.sativum_wa1_contig19033 & V-type proton ATPase subunit E & 0 & 0 & transport & $\mathrm{T} / \mathrm{V}$ & 8.0 & 2.7 & 6.0 & 4.7 & 0.6 & 1.7 & 1.3 & 0.4 & 0.3 & 0.8 & -0.0281 \\
\hline contig10734 & SDH flavoprotein subunit 1 & 0 & 0 & TCA cycle & $\mathrm{M}$ & 2.0 & 6.7 & 1.7 & 10.0 & 0.7 & 0.2 & 0.2 & 4.0 & 3.3 & 0.8 & -0.0313 \\
\hline contig20456 & HSP $70 \mathrm{kDa}$ & 0 & 0 & protein folding & $\mathrm{M}$ & 0.5 & 3.5 & 0.5 & 4.3 & 0.8 & 0.1 & 0.1 & 7.0 & 7.1 & 1.0 & -0.0558 \\
\hline p.sativum_wa1_contig16648 & ferredoxin & 0 & 0 & electron transfer & $\mathrm{P} / \mathrm{M}$ & 4.3 & 1.0 & 3.3 & 5.0 & 0.2 & 0.9 & 0.7 & 0.3 & 0.2 & 0.8 & -0.1138 \\
\hline Pisum_sativum_v2_Contig2233 & TOM40 homolog 1 & 1 & 1 & protein targeting & $\mathrm{M}$ & 0.5 & 1.3 & 0.5 & 8.3 & 0.2 & 0.1 & 0.1 & 2.7 & 2.6 & 1.0 & -0.1632 \\
\hline \multirow[t]{3}{*}{ Q43877 } & Duplicate protein - HMG-I/Y & 0 & 0 & not assigned & $\mathrm{N}$ & 0.5 & 0.5 & 6.0 & 5.3 & 0.1 & 0.1 & 1.1 & 0.1 & 1.0 & 10.0 & -0.1842 \\
\hline & & & & & & & \multirow{2}{*}{\multicolumn{3}{|c|}{$\begin{array}{l}\text { Significant fol } \\
\text { change }\end{array}$}} & 36 & 31 & 20 & 31 & 29 & 23 & \\
\hline & & & & & & & & & & 41 & 46 & 36 & 42 & 37 & 29 & \\
\hline
\end{tabular}




\section{Discussion}

Results once again displayed the difficulty of proteomic approaches for membrane proteins. Only $20 \%$ of the significantly changed proteins had a transmembrane region (Figure $2 \mathrm{~B}$ ) and a major accumulation of ribosomes was observed. This might be due to cytosolic contamination with ribosomal proteins, or it might be caused by the interaction with the (rough) endoplasmic reticulum, as previously discussed [29]. Other reasons might be the interaction with membrane proteins, e.g., during folding processes. Ribosomal proteins represent higher abundant proteins and are usually easily identified in microsomal fractions, which has been known for a relatively long time and was not only restricted to plant samples [17,30-33]. In general, it has been shown that a high plasticity exists in ribosomal protein regulation that is part of adaptive processes to differential stress or developmental aspects $[32,33]$. Furthermore, the chosen centrifugation speeds for the preparation of the microsomal enrichment, which were lower than for the usual microsomal preparations, might have resulted in a higher amount of cellular compartments, which might also present a lower coverage of membrane proteins. However, this does not influence the results on the proteins and how they relate to the root phenotype.

In this study, we found that morphological root phenotypes of four diverse pea cultivars are likely to be explained by their differential microsomal root proteomes. Vroege had the significantly shortest, and Kelvedon longest roots, respectively, and compared to the other cultivars (Figure 1B). Morphology of these four cultivars have been observed in earlier studies for older plants grown in hydroponics, showing that root length was only slightly different between cultivars, with Blauwschokker and Kelvedon exhibiting the longest roots [17]. If the differences in root length are due to different growth or differentiation, as well as the aspect of different hormones, should be investigated in the future.

Cultivar Kelvedon has previously been observed to be highly efficient in iron-deficient and high $\mathrm{pH}$ conditions [17,34]. Kelvedon is also resistant to pea wilt and tolerant to downy mildew (in reference to different breeders e.g., Tompson and Morgan, and the Royal Horticulture Society, www.rhs.org.uk). The latest data also suggest a higher Cd tolerance of Kelvedon [35]. Furthermore, a significant increase of $\mathrm{Cd}$ in roots, but not in leaves, was observed due to $\mathrm{Cd}$ stress. To date, cultivar Kelvedon has been shown in multiple studies to be superior in heavy metal-related stresses to other cultivars, and it would be interesting to see how this traditional cultivar is affected by other stresses, e.g., cold and water stress, and the reason for it. This will be an important step to breed more resistant cultivars in the future. In contrast, earlier studies have described Vroege to be relatively sensitive to iron deficiency conditions [17].

The generally increased protein levels, along with the root growth characteristics found at day five after germination, described above, might display a connection between increased stress susceptibility and a short root morphology. It is well known that enhanced root length can increase drought stress tolerance [36,37]. Determining if this trait is also involved in the enhancement of other stresses needs further investigation.

Altogether, 60 root microsomal proteins were found differentially abundant in the four cultivars after five days of germination (Supplemental Tables 1 and 2). The PCA analysis underpinned that several of these proteins might be involved in the different root phenotypes of the four cultivars (Figure 2A). Nevertheless, it remains unclear whether the difference was cause or the consequence of the developmental disparities.

The discussion is focused on those proteins appearing to impact most on the separation between the root phenotypes, which are potentially good markers. Interestingly, annexin-like protein RJ4 tagged to the nucleus showed the highest impact on the differentiation between the cultivars' root phenotypes. Annexins and annexin-like proteins are regularly found in proteomic analysis of membrane fractions [38,39]. They have a function in calcium-dependent phospholipid binding and are involved in root and nodule development [39-41]. Baucher et al. [42] described a tobacco annexin (Ntann 12) mainly localized in the nucleus of the root cells. The accumulation of Ntann 12 was strongly linked to auxin transport and signalling, which is also well known to play an essential role during root development [43,44]. In case of high auxin concentrations, root elongation is inhibited [45]. In addition, 
auxin accumulation in root cells was described to induce annexin Ntann12 [42]. In this study, annexin accumulation, therefore, implies that auxin levels were also increased in roots of cultivar Vroege. Hence, annexin-like protein RJ4 found in our study seems to be a good marker for hampered root development. Its accumulation might also reflect an early stage of development. The latter option may be aided by the finding of another putative marker, convicilin, solely enhanced in cultivar Vroege. Convicilin is a seed storage protein involved in seed development that was found earlier and described for pea [46]. It has been shown before that, in the pea embryonic axis, the storage proteins, including convicilin, changed during germination [11]. Its accumulation in cultivar Vroege underlines its earlier stage of seed development. Hence, the combination of the two putative marker proteins might be important to distinguish between developmental stages and root growth performance. However, this needs to be further analysed in the future.

As another interesting candidate, endoplasmin is a molecular chaperone of the heat-shock protein 90 class located in the endoplasmic reticulum [47], which plays a role in folding and is a highly regulated protein family. Due to the importance of endoplasmins for different stress conditions and developmental stages, these proteins have been found often regulatory relevant in proteomic studies [48,49]. Here, the strong induction of this protein (contig17941), together with several ribosomal proteins, implies an impairment in protein synthesis pathways leading to reduced root growth.

In contrast to the above-discussed putative markers for reduced root growth, our data provide evidence for a mitochondrial marker, TOM40 homolog 1, indicating enhanced root development. TOM40 is a central component of a hydrophilic channel of the mitochondrial outer membrane, the so-called translocase of the outer membrane (TOM), functioning as a protein import pore [50]. Thus, it is proposed that TOMs are directly linked with precursor proteins to participate in early stages of mitochondrial protein import. Mitochondria play an important role in energy-, redox-, and metabolic homeostasis, and have been shown to be integrated with plant growth regulation. Most mitochondrial mutants revealed reduced growth rates and have a short root phenotype [51]. Even though it is not clear how the TOM machinery might control the root development, elevated TOM40 levels appeared to be associated with increased root growth. This is also supported by the fact that other mitochondrial membrane markers were not significantly changed between the cultivars.

Several other proteins that significantly differentiate between the cultivars, such as proteins involved in transport, amino acids and redox-related proteins do not show an impact on the separation between the root phenotypes, but may play a role in other developmental regulations that cannot be unravelled in this study.

\section{Conclusions}

Summarizing, proteomic approaches have the potential to be used as alternative techniques to QTL mapping. Here, our shotgun proteomics approach enabled distinguishing different pea cultivars according to their root microsomal proteome linked to their root phenotypes. In contrast to genes, proteins represent the last instance for molecular reactions and the proteomic approach are complementary in finding implications between phenotypes, genotypes, and specific trades breeders are looking for. The analysed pea cultivars revealed microsomal proteins with high impacts on root development at the seedling stage possibly important for many other plants. We found the mitochondrial channel protein TOM40 to be most promising as a putative marker for improved root growth, which will be further investigated in the future. In contrast, the accumulation of an annexin-like protein RJ4 and convicilin seemed to be a potential marker associated with shortened root growth.

Supplementary Materials: Supplementary materials are available online.

Acknowledgments: This work was supported by the Young Researcher Initiative of the University of Hamburg (Post-Doc grant to CM). The authors have declared no conflict of interest. 
Author Contributions: C.N. Meisrimler conceived and designed the experiments. C.N. Meisrimler and S. Wienkoop performed the experiments and analyzed the data. S. Lüthje contributed reagents/materials/analysis tools. C.N. Meisrimler was supported by the two other authors. The manuscript was written by all three authors.

Conflicts of Interest: The authors declare no conflict of interest.

\section{References}

1. Smýkal, P.; Aubert, G.; Burstin, J.; Coyne, C.J.; Ellis, N.T.H.; Flavell, A.J.; Ford, R.; Hýbl, M.; Macas, J.; Neumann, P.; et al. Pea (Pisum sativum L.) in the Genomic Era. Agronomy 2012, 2, 74-115. [CrossRef]

2. FAOSTAT. Available online: http://faostat3.fao.org/home/E (accessed on 1 September 2016).

3. Meisrimler, C.-N.; Menckhoff, L.; Kukavica, B.M.; Lüthje, S. Pre-fractionation strategies to resolve pea (Pisum sativum) sub-proteomes. Front Plant Sci. 2015, 6, 849. [CrossRef] [PubMed]

4. Cool Season Food Legume Database. Available online: https://www.coolseasonfoodlegume.org/ (accessed on 1 September 2016).

5. Wang, J.; Meng, Y.; Li, B.; Ma, X.; Lai, Y.; Si, E.; Yang, K.; Xu, X.; Shang, X.; Wang, H.; et al. Physiological and proteomic analyses of salt stress response in the halophyte Halogeton glomeratus. Plant Cell Environ. 2015, 38, 655-669. [CrossRef] [PubMed]

6. Meisrimler, C.N.; Planchon, S.; Renaut, J.; Sergeant, K.; Lüthje, S. Alteration of plasma membrane-bound redox systems of Fe deficient pea roots by chitosan. J. Proteom. 2011, 74, 1437-1449. [CrossRef] [PubMed]

7. Vincent, D.; Ergül, A.; Bohlman, M.C.; Tattersall, E.A.; Tillett, R.L.; Wheatley, M.D.; Woolsey, D.R.; Quilici, D.R.; Joets, J.; Schlauch, K.; et al. Proteomic analysis reveals differences between Vitis vinifera L. cv. Chardonnay and cv. Cabernet Sauvignon and their responses to water deficit and salinity. J. Exp. Bot. 2011, 58, 1873-1892. [CrossRef] [PubMed]

8. $\quad$ Stark, A.L.; Hause, R.J., Jr.; Gorsic, L.K.; Antao, N.N.; Wong, S.S.; Chung, S.H.; Daniel, F.G.; Im, H.K.; Myers, J.L.; White, K.P.; et al. Protein quantitative trait loci identify novel candidates modulating cellular response to chemotherapy. PLoS Genet. 2014, 10, e1004192. [CrossRef] [PubMed]

9. Castillejo, M.Á.; Iglesias-García, R.; Wienkoop, S.; Rubiales, D. Label-free quantitative proteomic analysis of tolerance to drought in Pisum sativum. Proteomics 2016, 16, 2776-2787. [CrossRef] [PubMed]

10. Jain, A.; Singh, A.; Singh, S.; Singh, V.; Singh, H.B. Comparative proteomic analysis in pea treated with microbial consortia of beneficial microbes reveals changes in the protein network to enhance resistance against Sclerotinia sclerotiorum. J. Plant Physiol. 2015, 182, 79-94. [CrossRef] [PubMed]

11. Wang, W.Q.; Møller, I.M.; Song, S.Q. Proteomic analysis of embryonic axis of Pisum sativum seeds during germination and identification of proteins associated with loss of desiccation tolerance. J. Protoc. 2012, 77, 68-86. [CrossRef] [PubMed]

12. Badowiec, A.; Swigonska, S.; Weidner, S. Changes in the protein patterns in pea (Pisum sativum L.) roots under the influence of long- and short-term chilling stress and post-stress recovery. Plant Phys. Biochem. 2013, 71, 315-324. [CrossRef] [PubMed]

13. Nacry, P.; Canivenc, G.; Muller, B.; Azmi, A.; Van Onckelen, H.; Rossignol, M.; Doumas, P. A role for auxin redistribution in the responses of the root system architecture to phosphate starvation in Arabidopsis. Plant Physiol. 2005, 138, 2061-2074. [CrossRef] [PubMed]

14. Lee, S.; Lee, E.J.; Yang, E.J.; Lee, J.E.; Park, A.R.; Song, W.H.; Park, O.K. Proteomic identification of annexins, calcium-dependent membrane binding proteins that mediate osmotic stress and abscisic acid signal transduction in Arabidopsis. Plant Cell 2004, 16, 1378-1391. [CrossRef] [PubMed]

15. McPhee, K. Variation for seedling root architecture in the core collection of pea germplasm. Crop Sci. 2005, 45, 1758-1763. [CrossRef]

16. Shabalina, S.A.; Spiridonov, N.A.; Kashina, A. Sounds of silence: synonymous nucleotides as a key to biological regulation and complexity. Nucleic Acids Res. 2013, 41, 2073-2094. [CrossRef] [PubMed]

17. Meisrimler, C.N.; Wienkoop, S.; Lyon, D.; Geilfus, C.M.; Lüthje, S. Long-term iron deficiency: Tracing changes in the proteome of different pea (Pisum sativum L.) cultivars. J. Proteom. 2016, 140, 13-23. [CrossRef] [PubMed]

18. Bradford, M.M. A rapid and sensitive method for the quantitation of microgram quantities of protein utilizing the principle of protein-dye binding. Anal. Biochem. 1976, 72, 248-254. [CrossRef] 
19. Staudinger, C.; Mehmeti, V.; Turetschek, R.; Lyon, D.; Egelhofer, V.; Wienkoop, S. Possible Role of Nutritional Priming for Early Salt and Drought Stress Responses in Medicago truncatula. Front Plant Sci. 2012, 3, 285. [CrossRef] [PubMed]

20. Sun, Y.; Fleming, R.M.T.; Thiele, I.; Saunders, M.A. Robust flux balance analysis of multiscale biochemical reaction networks. BMC Bioinf. 2013, 14, 240. [CrossRef] [PubMed]

21. Lohse, M.; Nagel, A.; Herter, T.; May, P.; Schroda, M.; Zrenner, R.; Tohge, T.; Fernie, A.R.; Stitt, M.; Usadel, B. Mercator: A fast and simple web server for genome scale functional annotation of plant sequence data. Plant Cell Environ. 2014, 37, 1250-1258. [CrossRef] [PubMed]

22. Turetschek, R.; Lyon, D.; Desalegn, G.; Kaul, H.P.; Wienkoop, S. A Proteomic Workflow Using High-Throughput De Novo Sequencing Towards Complementation of Genome Information for Improved Comparative Crop Science. In Proteomis in Systems Biology SE - 17 Methods in Molecular Biology; Reinders, J., Ed.; Springer: New York, NY, USA, 2016; pp. 233-243.

23. Egelhofer, V.; Hoehenwarter, W.; Lyon, D.; Weckwerth, W.; Wienkoop, S. Using ProtMAX to Create High-Mass-Accuracy Precursor Alignments from Label-Free Quantitative Mass Spectrometry Data Generated in Shotgun Proteomics Experiments. Nat. Protoc. 2013, 8, 595-601. [CrossRef] [PubMed]

24. TMHMM. Available online: http://www.cbs.dtu.dk/services/TMHMM/ (accessed on 1 September 2016).

25. HMMTOP. Available online: http://www.enzim.hu/hmmtop/ (accessed on 1 January 2017).

26. Chou, K.C.; Shen, H.B. Plant-mPLoc: A top-down strategy to augment the power for predicting plant protein subcellular localization. PLoS ONE 2010, 5, e11335. [CrossRef] [PubMed]

27. Chou, K.C.; Shen, H.B. Cell-PLoc: A package of web-servers for predicting subcellular localization of proteins in various organisms. Nat. Protoc. 2008, 3, 153-162. [CrossRef] [PubMed]

28. Chou, K.C.; Shen, H.B. Large-Scale Plant Protein Subcellular Location Prediction. J. Cell. Biochem. 2007, 100, 665-678. [CrossRef] [PubMed]

29. Freedman, S.D.; Jamieson, J.D. Hormone-induced protein phosphorylation. II. Localization to the ribosomal fraction from rat exocrine pancreas and parotid of a 29,000-dalton protein phosphorylated in situ in response to secretagogues. J. Cell. Biol. 1982, 95, 909-917. [CrossRef] [PubMed]

30. Koldamova, R.P.; Lefterov, I.M.; DiSabella, M.T.; Almonte, C.; Watkins, S.C.; Lazo, J.S. Human bleomycin hydrolase binds ribosomal proteins. Biochemistry 1999, 38, 7111-7117. [CrossRef] [PubMed]

31. Zargar, S.M.; Kurata, R.; Inaba, S.; Oikawa, A.; Fukui, R.; Ogata, Y.; Agrawal, G.K.; Rakwal, R.; Fukao, Y. Quantitative proteomics of Arabidopsis shoot microsomal proteins reveals a cross-talk between excess zinc and iron deficiency. Proteomics 2015, 15, 1196-1201. [CrossRef] [PubMed]

32. Wang, J.; Lan, P.; Gao, H.; Zheng, L.; Li, W.; Schmidt, W. Expression changes of ribosomal proteins in phosphate-and iron-deficient Arabidopsis roots predict stress-specific alterations in ribosome composition. BMC Genom. 2013, 14, 1. [CrossRef] [PubMed]

33. Lan, P.; Schmidt, W. The enigma of eIF5A in the iron deficiency response of Arabidopsis. Plant Sign. Behav. 2011, 6, 528-530. [CrossRef]

34. Jelali, N.; Moez, S.; Dhifi, W.; Mnif, W.; Abdelly, C.; Gharsalli, M. Secondary metabolism responses in two Pisum sativum L. cultivars cultivated under Fe deficiency conditions. Afr. J. Biotech. 2012, 11, 14828-14836.

35. Rahman, M.F.; Islam, M.; Begum, M.C.; Kabir, A.H.; Alam, M.F. Genetic variation in cadmium tolerance is related to transport and antioxidant activities in field peas (Pisum sativum L.). Arch. Agron. Soil Sci. 2016, 1-8. [CrossRef]

36. Comas, L.H.; Becker, S.R.; von Mark, V.C.; Byrne, P.F.; Dierig, D. Root traits contributing to plant productivity under drought. Front. Plant Sci. 2013, 4, 442. [CrossRef] [PubMed]

37. Chimungu, J.G.; Brown, K.M.; Lynch, J.P. Large root cortical cell size improves drought tolerance in maize. Plant Physiol. 2014, 166, 2166-2178. [CrossRef] [PubMed]

38. Nie, L.; Feng, J.; Fan, P.; Chen, X.; Guo, J.; Lv, S.; Bao, H.; Jia, W.; Tai1, F.; Jiang, P.; et al. Comparative proteomics of root plasma membrane proteins reveals the involvement of calcium signalling in $\mathrm{NaCl}$-facilitated nitrate uptake in Salicornia europaea. J. Exp. Bot. 2015, erv216. [CrossRef]

39. Wienkoop, S.; Saalbach, G. Proteome analysis. Novel proteins identified at the peribacteroid membrane from Lotus japonicus root nodules. Plant Physiol. 2003, 131, 1080-1090. [CrossRef] [PubMed]

40. Talukdar, T.; Gorecka, K.M.; de Carvalho-Niebel, F.; Downie, J.A.; Cullimore, J.; Pikula, S. Annexins-Calcium- and membrane-binding proteins in the plant kingdom. Potential role in nodulation and mycorrhization in Medicago truncatula. Acta Biochim. Pol. 2009, 56, 199-210. [PubMed] 
41. Lino, B.; Chagolla, A.; de la Vara, L.G. Membrane proteins involved in transport, vesicle traffic and Ca ${ }^{2+}$ signaling increase in beetroots grown in saline soils. Planta 2016, 244, 87-101. [CrossRef] [PubMed]

42. Baucher, M.; Lowe, Y.O.; Vandeputte, O.M.; Bopopi, J.M.; Moussawi, J.; Vermeersch, M.; Mol, A.; El Jaziri, M.; Homblé, F.; Pérez-Morga, D. Ntann12 annexin expression is induced by auxin in tobacco roots. J. Exp. Bot. 2011, 62, 4055-4065. [CrossRef] [PubMed]

43. Overvoorde, P.; Fukaki, H.; Beeckman, T. Auxin control of root development. Cold Spring Harb. Perspect. Biol. 2010, 2, 1-16. [CrossRef] [PubMed]

44. Saini, S.; Sharma, I.; Kaur, N.; Pati, P.K. Auxin: A master regulator in plant root development. Plant Cell Rep. 2013, 32, 741-757. [CrossRef] [PubMed]

45. James, B.; Koepfli, J.B. The Inhibition of Root Growth by Auxins. Am. J. Bot. 1939, 26, 557-566.

46. Croy, R.R.; Gatehouse, J.A.; Evans, I.M.; Boulter, D. Characterisation of the storage protein subunits synthesised in vitro by polyribosomes and RNA from developing pea (Pisum sativum L.). Planta 1980, 148, 49-56. [CrossRef] [PubMed]

47. Klein, E.M.; Mascheroni, L.; Pompa, A.; Ragni, L.; Weimar, T.; Lilley, K.S.; Dupree, P.; Vitale, A. Plant endoplasmin supports the protein secretory pathway and has a role in proliferating tissues. Plant J. 2006, 48, 657-673. [CrossRef] [PubMed]

48. Qian, D.; Tian, L.; Qu, L. Proteomic analysis of endoplasmic reticulum stress responses in rice seeds. Sci. Rep. 2015, 5, 14255. [CrossRef] [PubMed]

49. Li, W.; Wei, Z.; Qiao, Z.; Wu, Z.; Cheng, L.; Wang, Y. Proteomics Analysis of Alfalfa Response to Heat Stress. PLoS ONE 2013, 8, e82725. [CrossRef] [PubMed]

50. Hill, K.; Model, K.; Ryan, M.T.; Dietmeier, K.; Martin, F.; Wagner, R.; Pfanner, N. Tom40 forms the hydrophilic channel of the mitochondrial import pore for preproteins. Nature 1998, 395, 516-521. [PubMed]

51. Hsieh, W.Y.; Liao, J.C.; Hsieh, M.H. Dysfunctional mitochondria regulate the size of root apical meristem and leaf development in Arabidopsis. Plant Signal. Behav. 2015, 10, e1071002. [CrossRef] [PubMed]

(c) 2017 by the authors. Licensee MDPI, Basel, Switzerland. This article is an open access article distributed under the terms and conditions of the Creative Commons Attribution (CC BY) license (http:/ / creativecommons.org/licenses/by/4.0/). 